\title{
ChemComm
}

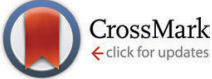

Cite this: Chem. Commun., 2015, 51,12463

Received 6th June 2015,

Accepted 28th June 2015

DOI: $10.1039 / c 5 c c 04663 g$

www.rsc.org/chemcomm

\section{Hybrid materials of Ni NPaMOF prepared by a simple synthetic method $\dagger$}

\author{
Megumi Mukoyoshi, ${ }^{a}$ Hirokazu Kobayashi, ${ }^{\text {ab }}$ Kohei Kusada, ${ }^{a b}$ Mikihiro Hayashi, ${ }^{a}$ \\ Teppei Yamada, $\neq^{a}$ Mitsuhiko Maesato, ${ }^{a}$ Jared M. Taylor, ${ }^{a b}$ Yoshiki Kubota, ${ }^{c}$ \\ Kenichi Kato, de Masaki Takata, de Tomokazu Yamamoto, ${ }^{\text {bf }}$ Syo Matsumurabfg and \\ Hiroshi Kitagawa*abhi
}

We demonstrate a novel, simple synthetic method for metal (Ni) NPs in a MOF using the partial thermal decomposition of nickel(II) 2,5-dihydroxyterephthalate (Ni-MOF-74). The Ni NPs inside the Ni-MOF-74 are several nanometers in size, and the size can be precisely controlled by the heating conditions.

Metal-organic frameworks (MOFs), which consist of organic ligands connecting metal ions into porous crystalline framework structures, have garnered significant interest because of the versatile nature of their structures. ${ }^{1-3}$ Their high structural versatility allows for a wide range of applications such as gas storage, ${ }^{4-6}$ separations ${ }^{7,8}$ and ion conduction, ${ }^{9,10}$ which arise from the porous nature of the MOFs and are optimized based on the organic ligands and metal ions which comprise the MOFs. This pore space can also be utilized to create multifunctional composite materials by including nanoparticles

${ }^{a}$ Division of Chemistry, Graduate School of Science, Kyoto University,

Kitashirakawa-Oiwakecho, Sakyo-ku, Kyoto 606-8502, Japan.

E-mail: hkobayashi@kuchem.kyoto-u.ac.jp,kitagawa@kuchem.kyoto-u.ac.jp

${ }^{b}$ JST CREST, 7, Gobancho, Chiyoda-ku, Tokyo 102-0076, Japan

${ }^{c}$ Department of Physical Science, Graduate School of Science,

Osaka Prefecture University, Sakai, Osaka 599-8531, Japan

${ }^{d}$ RIKEN SPring-8 Center, 1-1-1 Kouto, Sayo-cho, Sayo-gun, Hyogo 679-5148, Japan

${ }^{e}$ Japan Synchrotron Radiation Research Institute, 1-1-1 Kouto, Sayo-cho, Sayo-gun, Hyogo 679-5198, Japan

${ }^{f}$ Department of Applied Quantum Physics and Nuclear Engineering,

Graduate School of Engineering, Kyushu University, Motooka 744, Nishi-ku,

Fukuoka 819-0395, Japan

${ }^{g}$ Research Laboratory for High-Voltage Electron Microscopy, Kyushu University, Motooka 744, Nishi-ku, Fukuoka 819-0395, Japan

${ }^{h}$ Institute for Integrated Cell-Material Sciences (iCeMS), Kyoto University, Yoshida, Sakyo-ku, Kyoto 606-8501, Japan

${ }^{i}$ INAMORI Frontier Research Center, Kyushu University, Motooka 744, Nishi-ku, Fukuoka 819-0395, Japan

$\dagger$ Electronic supplementary information (ESI) available: Experimental details, TG analysis, heat treatment, X-ray diffraction, Fourier-transform infrared spectroscopy, EDX maps, TEM images, nitrogen sorption and magnetic properties. See DOI: $10.1039 / \mathrm{c} 5 \mathrm{cc} 04663 \mathrm{~g}$

\$ Current addresses: Center for Molecular Systems (CMS), Kyushu University, Motooka 744, Nishi-ku, Fukuoka 819-0395, Japan; Department of Chemistry and Biochemistry, Graduate School of Engineering, Kyushu University, Motooka 744, Nishi-ku, Fukuoka 819-0395, Japan.
(NPs) to synergize with the properties of MOFs for material or energy conversion applications. ${ }^{11-15}$ The NP/MOF composite materials often show enhanced properties versus the pure materials due to such synergistic effects, so NP/MOF composites are a promising route to control or enhance the properties of NPs for a variety of applications. But while the synergistic properties of NP/MOF composite materials appear to be promising, more simple synthetic methods are required for widespread use.

Recently, various methods for preparing metal NP/MOF composites have been developed. The first attempt at loading MOFs with NPs was by simple mixing of the MOF and the metal precursor via solid grinding ${ }^{16,17}$ or solution infiltration, ${ }^{13,18}$ and the metal NP/MOFs were fabricated by reduction of the metal precursor. More recently, a chemical vapour deposition (CVD) method has been developed to better control the resultant NP size, whereby porous MOFs are loaded with a metal NP precursor in the vapour phase and then subsequently decomposed to create metal NPs within the MOF pores. ${ }^{11,19-21}$ This CVD technique is considered to be a complicated process that is not suited to large-scale production. Furthermore, a multistep process may be required for the preparation of volatile organometallic precursors which are typically unstable in air. Metal NP/MOF composites have also been prepared by encapsulating pre-synthesized metal NPs by growing the MOF around them, ${ }^{22-24}$ but a drawback of this method is that any protecting agents and/or surfactants used to synthesize the metal NPs are retained as impurities. We wanted to develop a method whereby we could generate metal NPs within the MOF by a simple method without complicated infiltration or growth procedures. Here, we report a novel, hybridization method by partial thermal decomposition of a MOF to generate metal NPs with tuneable size within the MOF (metal NP@MOF) (Fig. 1). For this study we used the well known Ni-MOF-74, ${ }^{25} \mathrm{Ni}_{2}$ (dhtp) ( $\mathrm{H}_{4} \mathrm{dhtp}=2,5$-dihydroxyterephthalic acid), for an initial demonstration. These hybrid materials contain metal NPs within a MOF without any additional protecting agents and/or surfactants because none are used (Fig. 1).

In order to generate a metal NP/MOF composite, we used Ni-MOF-74 because it has large, cylindrical, 1-dimensional pores 


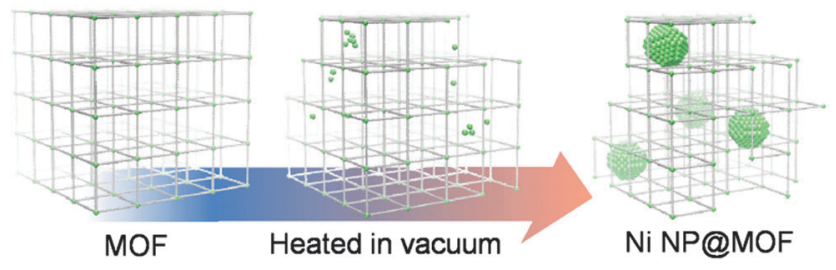

Fig. 1 Scheme of the synthesis of Ni NPs@MOF.

(diameter of $11 \AA^{25}$ ) which can act as functional pore space, and it is constructed from a combination of nickel(II) which can be reduced and a functionalized hydroqinone ligand, which can act as a reducing agent. Ni-MOF-74 was synthesized using a slight modification of a previously reported procedure, ${ }^{26}$ and the sample quality was checked using a combination of powder X-ray diffraction (PXRD), thermogravimetric analysis (TGA) and $\mathrm{N}_{2}$ gas sorption analysis. By TGA, the Ni-MOF-74 immediately begins losing included solvent, and decomposition occurs between 300 and $360{ }^{\circ} \mathrm{C}$ (Fig. S1, ESI $\dagger$ ). We hypothesized that the decomposition may result from electron transfer from the ligand to metal ion, so for partial decomposition, we heated the Ni-MOF-74 to temperatures between $250-350{ }^{\circ} \mathrm{C}$ under vacuum and obtained hybrid materials - Ni NPs contained within the Ni-MOF-74 (Ni NP@MOF-74) (Table S1, ESI $\dagger$ ). Fig. 2a shows the PXRD patterns of Ni-MOF-74 heated at various temperatures for $12 \mathrm{~h}$ under vacuum. The sample heated at $300{ }^{\circ} \mathrm{C}$ for $12 \mathrm{~h}(300-12 \mathrm{~h})$ yielded an identical diffraction pattern to Ni-MOF-74, but by increasing the temperature to $350{ }^{\circ} \mathrm{C}$ for $12 \mathrm{~h}$ (350-12h), broad peaks corresponding to a face-centered-cubic (fcc) Ni lattice appeared along with the Ni-MOF-74 pattern; increasing
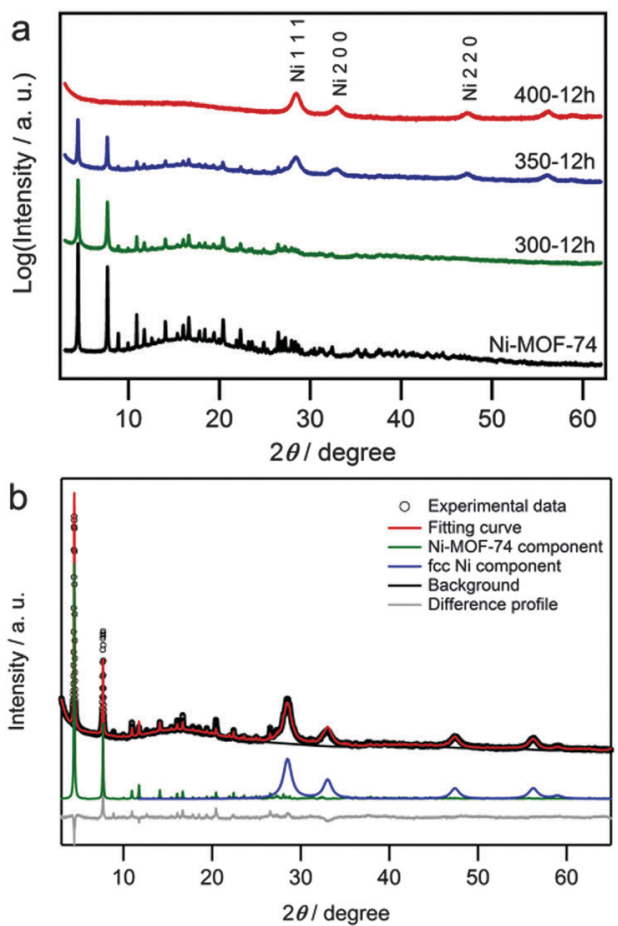

Fig. 2 (a) Powder XRD patterns of Ni-MOF-74 (black line), 300-12h (green line), $350-12 \mathrm{~h}$ (blue line) and 400-12h (red line). The radiation wavelength was $99.8 \mathrm{pm}$. (b) Rietveld analysis for 350-12h. the heating time increases the intensity of the fcc-Ni peaks (Fig. S2a-d, ESI $\dagger$ ). Higher temperature heat treatment at $400{ }^{\circ} \mathrm{C}$ (400-12h) caused complete elimination of the initial Ni-MOF-74 diffraction pattern, and only a fcc-Ni pattern was observed. An $a=3.52(1) \AA$ lattice constant was obtained using Rietveld refinement, which was a typical value for fcc-Ni NP. ${ }^{27}$ Furthermore, infrared (IR) spectra of samples $300-12 \mathrm{~h}$ and $350-12 \mathrm{~h}$ were very similar to that of Ni-MOF-74, ${ }^{28}$ which confirmed that Ni-MOF-74 was still present in these hybrid materials (Fig. S3, ESI $\dagger$ ). From the result of elemental analysis, the composition of Ni NP@Ni-MOF-74 was estimated to be $\mathrm{Ni}_{2}\left(\mathrm{C}_{8} \mathrm{O}_{6} \mathrm{H}_{2}\right) \cdot 9 \mathrm{H}_{2} \mathrm{O} \cdot 5 \mathrm{Ni} \cdot 6 \mathrm{C}$, showing that a significant portion of the ligand decomposes into volatile products, leaving a small amount of residual carbon.

Then, we performed Rietveld refinement on PXRD patterns from $350-12 \mathrm{~h}$ using the fixed position from the $\mathrm{Zn}$-MOF- $74^{29}$ cif file as a structural model for the Ni-MOF-74 component (Fig. 2b). Lattice constants were obtained for the two components: $a=25.928(4) \AA$ and $c=6.694(2) \AA$ for the MOF- 74 and $a=3.5236$ (7) $\AA$ for the Ni-fcc lattices (Fig. S2e, ESI $\dagger$ ). These lattice constants are consistent with those of each component, the $\mathrm{MOF}^{25}$ and Ni NPs, ${ }^{27}$ and the Ni crystal size was estimated to be 5.3(1) nm using the Scherrer formula.

Ni 2p X-ray photoelectron spectroscopy (XPS) was also performed to determine $\mathrm{Ni}$ oxidation states (Fig. 3). Two peaks in the Ni $2 p$ region of the XPS spectra for Ni-MOF-74 were observed centered at $856.1\left(2 \mathrm{p}_{3 / 2}\right)$ and $873.8\left(2 \mathrm{p}_{1 / 2}\right) \mathrm{eV}$, which corresponds to the $\mathrm{Ni}^{2+}$ ions ${ }^{30}$ in Ni-MOF-74. In addition to these peaks, samples $300-12 \mathrm{~h}$ and $350-12 \mathrm{~h}$ yielded a new pair of spin-orbit splitting peaks at $852.8\left(2 \mathrm{p}_{3 / 2}\right)$ and $870.0 \mathrm{eV}\left(2 \mathrm{p}_{1 / 2}\right)$, which correspond to the formation of Ni NPs. ${ }^{30}$ For $400-12 \mathrm{~h}$, only the Ni NP signals were observed, and the higher binding energy signals from the $\mathrm{Ni}^{2+}$ in Ni-MOF-74 were not observed, which is consistent with the PXRD results. These results strongly suggest that samples $300-12 \mathrm{~h}$ and 350-12h contained both the Ni NPs and the MOF.

We further characterized the samples after heat treatment at various temperatures using transmission electron microscopy (TEM). Fig. 4a shows the TEM image of sample 350-12h. Highly dispersed NPs were found to evolve and distribute throughout the MOF. The mean diameter was estimated to be $4.3 \pm 1.4 \mathrm{~nm}$, which is consistent with the crystal size estimated from the diffraction

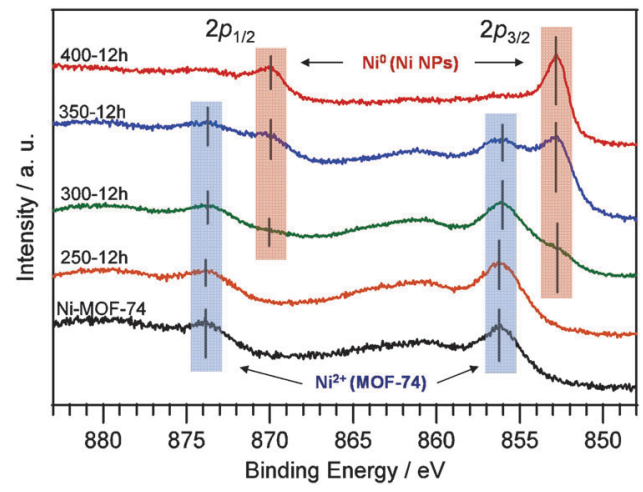

Fig. 3 The Ni 2p XPS spectra of Ni-MOF-74 (black line), 250-12h (orange line), 300-12h (green line), 350-12h (blue line) and 400-12h (red line). 


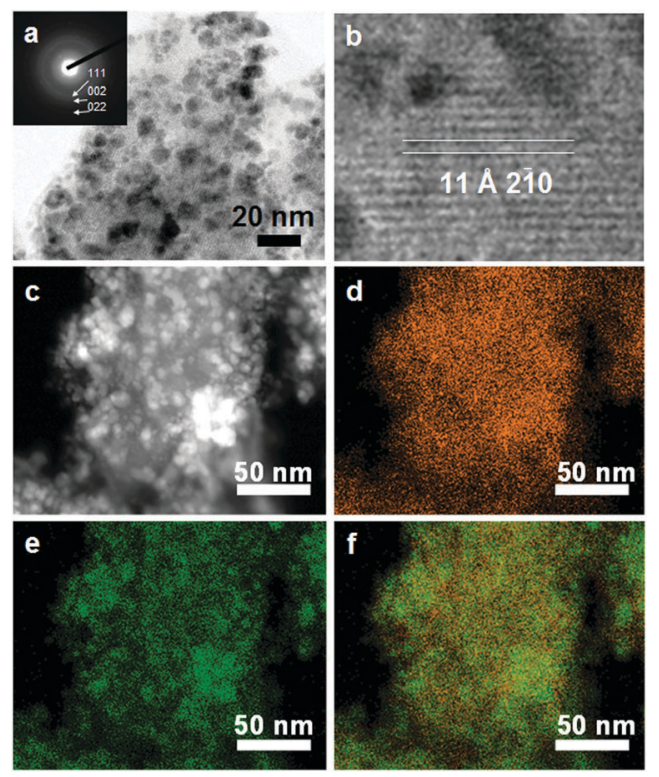

Fig. 4 (a) TEM image of 350-12h with electron diffraction (inset) and (b) the high magnification image. (c) HAADF-STEM image, (d) C-K STEM-EDX map (e) and Ni-K STEM-EDX map of 350-12h. (f) The reconstructed overlay image of the maps shown in (d) and (e).

pattern $(5.3 \mathrm{~nm})$. The HRTEM image indicated that Ni-MOF-74 had well-defined crystalline lattice fringes with an estimated lattice spacing of $11 \AA$, which corresponds to the Ni-MOF-74 (2-10) lattice plane (Fig. 4b). In addition, electron diffraction peaks originating from the fcc (111), (002) and (022) planes of the Ni NPs were observed (Fig. 4a inset). We further investigated the elemental distribution of the composite Ni NPs and Ni-MOF-74 (Fig. 4c-f). Fig. 4c shows a high-angle annular dark-field STEM (HAADF-STEM) image. Fig. 4d and e show the corresponding C-K and Ni-K STEMenergy dispersive X-ray (STEM-EDX) maps, respectively. The elemental C corresponds to the constituent dhtp ligand in Ni-MOF-74. Fig. 4f overlays the $\mathrm{C}$ and Ni elemental distributions. Compared to the Ni-MOF-74 mapping data (Fig. S4a-d, ESI $\dagger$ ), these mapping data show that the $\mathrm{C}$ in Ni-MOF-74 was distributed around the Ni NP surfaces. From these results, it was concluded that hybrid Ni NP materials were successfully synthesized within Ni-MOF-74.

The rich redox activity of $\mathrm{H}_{4} \mathrm{dhtp}$ (2,5-dihydroxyterephthalic acid) is notable in that the substituent hydroqinone ${ }^{31}$ plays a key role in reducing $\mathrm{Ni}^{2+}$ ions and generating the Ni NPs. For quinonebased metal complexes, it is well known that strong electron transfer from the ligand to metal ion occurs. ${ }^{32}$ Therefore, the dhtp ligand is considered to reduce the $\mathrm{Ni}^{2+}$ ion in our case with an accompanying cleavage of the Ni-O bond via the thermal decomposition of Ni-MOF-74. In addition, a semiquinone-like dhtp radical would be generated after this electron transfer. This unstable reaction intermediate is expected to promote the further reduction of $\mathrm{Ni}$ ions to form the more stable quinone. These reactions might be a reasonable driving force for creating the $\mathrm{Ni}$ NPs in MOF-74 via heat treatment.

The synthesis of several nanometer-scale Ni particles is still challenging because the Ni NPs are easily oxidized and aggregate during particle growth. Our work provides a simple and effective synthetic method for not only highly dispersed Ni@MOF hybrid materials but also Ni particles several nanometres in size. The mean diameter of the Ni NPs can be precisely controlled at the nanometre level from $2.0 \mathrm{~nm}$ to $5.0 \mathrm{~nm}$ by adjusting the heating conditions, such as the temperature and/or time (Fig. S5 and Table S2, ESI $\dagger$ ).

In order to obtain the synergistic properties of the nanoparticle and MOF, the nanoparticles need to remain accessible to guest species in the MOF. In order to investigate that the MOF still retained its important porous properties, $\mathrm{N}_{2}$ adsorption/desorption isotherms were measured at $77 \mathrm{~K}$ for Ni-MOF-74 and Ni NP@NiMOF-74 (Fig. 5). For Ni-MOF-74, the type I isotherm shape is a result of the microporosity of this $\mathrm{MOF} .^{25}$ After the heat treatment, the $\mathrm{N}_{2}$ absorption decreased, which indicates that there is partial loss of the MOF-74 micropores accompanying the generation of $\mathrm{Ni}$ NPs. Isotherms of samples heated at different treatment times support these results (Fig. S6, ESI $\dagger$ ). The adsorption/desorption hysteresis loop at $c a$. $0.6 P / P_{0}$ for the $300-12 \mathrm{~h}$ sample indicates the development of mesoporosity. ${ }^{33}$ The calculated BET surface areas for the initial Ni-MOF-74, 300-12h, 350-12h and 400-12h samples were $1280,1230,765$ and $163 \mathrm{~m}^{2} \mathrm{~g}^{-1}$, respectively. These values indicate that while porosity decreases upon the generation of $\mathrm{Ni}$ NPs due to partial framework decomposition to form dense Ni NPs, the MOF surrounding the NPs in the 350-12h sample still remains intact and highly porous.

Magnetic nanoparticles with a single magnetic domain are well known to show superparamagnetism above the blocking temperature, $T_{\mathrm{B}}$, and $T_{\mathrm{B}}$ is sensitive to the particle size. Fig. 6a shows the temperature dependencies of the zero-field-cooled (ZFC) and field-cooled (FC) magnetization for 300-12h. As shown in Fig. 6a, $300-12 \mathrm{~h}$ showed both antiferromagnetic and superparamagnetic behavior because of the coexistence of Ni-MOF-74 and Ni NP. The small contribution of superparamagnetism is superimposed on the antiferromagnetic behavior of pristine Ni-MOF-74 at $15 \mathrm{~K}$ (Fig. S7, $\mathrm{ESI} \dagger$ ). ${ }^{34}$ The slightly S-shaped magnetization curve at $2 \mathrm{~K}$ (Fig. 6b) and the difference between the ZFC and FC curves below the $T_{\mathrm{B}}$, $\sim 20 \mathrm{~K}$, indicate that Ni NP were present, while $T_{\mathrm{B}}$ for $400-12 \mathrm{~h}$ was $\sim 90 \mathrm{~K}$ (Fig. S8, ESI $\dagger$ ). The lower $T_{\mathrm{B}}$ in $300-12 \mathrm{~h}$ indicates a smaller NP size than for 400-12h, which agrees well with the TEM observations. Thus, the superparamagnetic behaviour of Ni NPs is retained

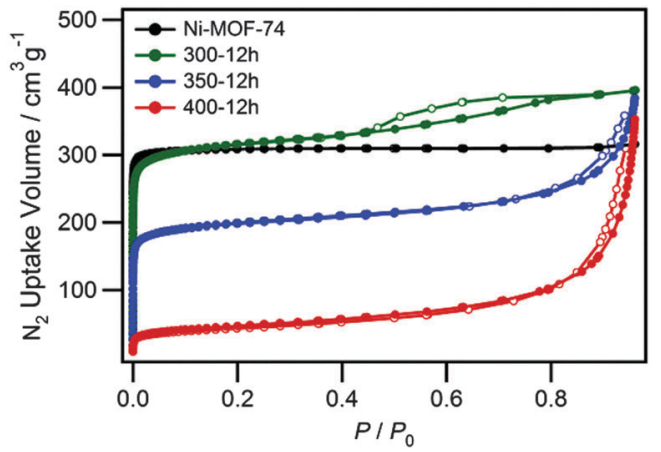

Fig. 5 Nitrogen sorption isotherms for Ni-MOF-74 (black circle), 300-12h (green circle), 350-12h (blue circle) and 400-12h (red circle) at $77 \mathrm{~K}$. The solid and open symbols represent adsorption and desorption, respectively. 

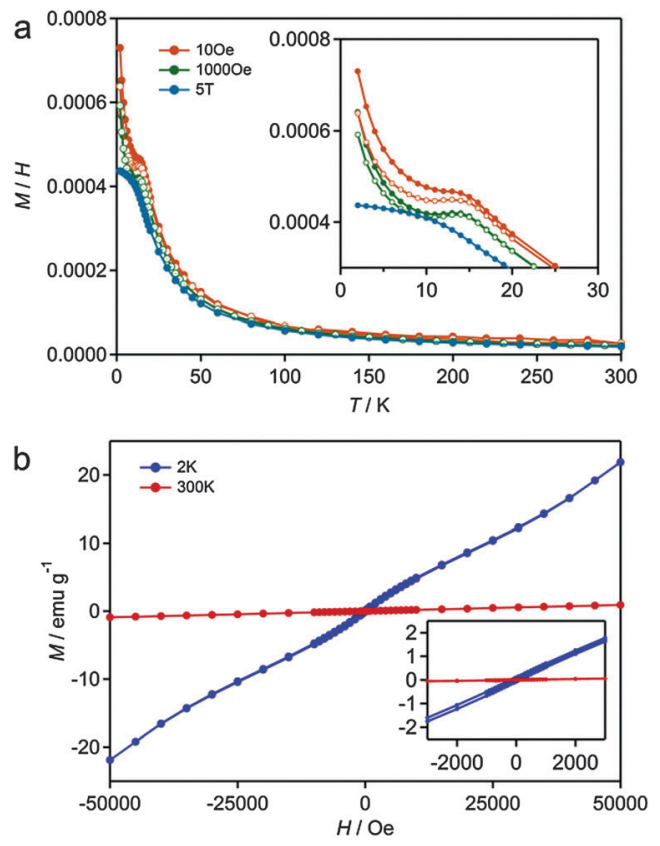

Fig. 6 (a) The magnetic susceptibility as a function of temperature for 300-12h (the open and solid symbols represent zero-field-cooled (ZFC) and field-cooled (FC), respectively). (b) Magnetization as a magnetic field for 300-12h at $2 \mathrm{~K}$ (blue circle) and $303 \mathrm{~K}$ (red circle).

for the hybrid materials, and the nucleation and successive growth of the NPs were confirmed by these magnetic measurements.

In summary, we developed a novel, simple synthetic method for metal Ni@MOF composites using the partial thermal decomposition of Ni-MOF-74. Furthermore, this method was effective for fabricating the metal NP@MOF composites, and by tuning the temperature conditions the size of the metal NP was controlled. The obtained Ni NP@Ni-MOF-74 composites possessed characteristics derived from both the Ni-MOF-74, such as porosity and antiferromagnetism, combined with the superparamagnetic properties of the isolated Ni NPs. As Ni is well known as an important catalyst, these hybrid materials have the potential to form effective catalysts with high activity and selectivity, resulting from the synergistic effects of the several nanometer-scale $\mathrm{Ni}$ catalyst and porous properties of the MOF. This approach provides a novel and simple method for the generation of a diverse class of metal nanoparticle composites, as analogous MOF-74 materials composed of Co, Mn, $\mathrm{Fe}, \mathrm{Zn}$ or $\mathrm{Mg}$ as a metal cation exist, and may be important for the future development of multi-functional materials.

The synchrotron XRD measurements were supported by the Japan Synchrotron Radiation Research Institute (JASRI) (Proposal No. 2012B1518). HRTEM, HAADF-STEM and EDX analyses were performed partly as a research program (A-13-KU-0100) of the Nanotechnology Platform project conducted by MEXT.

\section{Notes and references}

1 S. Kitagawa, R. Kitaura and S. Noro, Angew. Chem., Int. Ed., 2004, 43, 2334-2375.

2 J. R. long and O. M. Yaghi, Chem. Soc. Rev., 2009, 38, 1213-1214.
3 O. M. Yaghi, M. O'Keeffe, N. W. Ockwig, H. K. Chae, M. Eddaoudi and J. Kim, Nature, 2003, 423, 705-714.

4 M. Eddaoudi, J. Kim, N. Rosi, D. Vodak, J. Wachter, M. O'Keeffe and O. M. Yaghi, Science, 2002, 295, 469-472.

5 N. Rosi, J. Eckert, M. Eddaoudi, D. Vodak, J. Kim, M. O'Keeffe and O. M. Yaghi, Science, 2003, 300, 1127-1129.

6 H. Furukawa, N. Ko, Y. B. Go, N. Aratani, S. B. Choi, E. Choi, A. O. Yazaydin, R. Q Snurr, M. O'Keeffe, J. Kim and O. M. Yaghi, Science, 2010, 329, 424-428.

7 J. R. Li, R. J. Kuppler and H. C. Zhou, Chem. Soc. Rev., 2009, 38, 1477-1504.

8 O. M. Yaghi, G. Li and H. Li, Nature, 1995, 378, 703-706.

9 M. Sadakiyo, T. Yamada and H. Kitagawa, J. Am. Chem. Soc., 2009, 131, 9906-9907.

10 J. A. Hurd, R. Vaidhyanathan, V. Thangadurai, C. I. Ratcliffe, I. L. Moudrakovski and G. K. H. Shimizu, Nat. Chem., 2009, 1, 705-710.

11 J. Hermannsdörfer, M. Friedrich, N. Miyajima, R. Q. Albuquerque, S. Kümmel and R. Kempe, Angew. Chem., Int. Ed., 2012, 51, 11473-11477.

12 Q. L. Zhu, J. Li and Q. Xu, J. Am. Chem. Soc., 2013, 135, 10210.

13 B. Yuan, Y. Pan, Y. Li, B. Yin and H. Jiang, Angew. Chem., Int. Ed., 2010, 49, 4054-4058.

14 D. W. Lim, J. W. Yoon, K. Y. Ryu and M. P. Suh, Angew. Chem., Int. Ed., 2012, 51, 9814-9817.

15 C. Zlotea, R. Campesi, F. Cuevas, E. Leroy, P. Dibandjo, C. Volkringer, T. Loiseau, G. R. Ferey and M. Latroche, J. Am. Chem. Soc., 2010, 132, 2991-2997.

16 T. Ishida, M. Nagaoka, T. Akita and M. Haruta, Chem. - Eur. J., 2008, 14, 8456-8460.

17 H.-L. Jiang, B. Liu, T. Akita, M. Haruta, H. Sakurai and Q. Xu, J. Am. Chem. Soc., 2009, 131, 11302-11303.

18 R. J. T. Houk, B. W. Jacobs, F. El Gabaly, N. N. Chang, A. A. Talin, D. D. Graham, S. D. House, I. M. Robertson and M. D. Allendorf, Nano Lett., 2009, 9, 3413-3418.

19 S. Hermes, M. K. Schroter, R. Schmid, L. Khodeir, M. Muhler, A. Tissler, R. W. Fischer and R. A. Fischer, Angew. Chem., Int. Ed., 2005, 44, 6237-6241.

20 Y. K. Park, S. B. Choi, H. J. Nam, D.-Y. Jung, H. C. Ahn, K. Choi, H. Furukawa and J. Kim, Chem. Commun., 2010, 46, 3086-3088.

21 P. Z. Li, K. Aranishi and Q. Xu, Chem. Commun., 2012, 48, 3173-3175.

22 G. Lu, S. Li, Z. Guo, O. K. Farha, B. G. Hauser, X. Qi, Y. Wang, X. Wang, S. Han, X. Liu, J. S. DuChene, H. Zhang, Q. Zhang, X. Chen, J. Ma, S. C. J. Loo, W. D. Wei, Y. Yang, J. T. Hupp and F. Huo, Nat. Chem., 2012, 4, 310-316.

23 P. Falcaro, A. J. Hill, K. M. Nairn, J. Jasieniak, J. I. Mardel, T. J. Bastow, S. C. Mayo, M. Gimona, D. Gomez, H. J. Whitfield, R. Ricco, A. Patelli, B. Marmiroli, H. Amenitsch, T. Colson, L. Villanova and D. Buso, Nat. Commun., 2011, 2, 237-244.

24 K. Khaletskaya, J. Reboul, M. Meilikhov, M. Nakahama, S. Diring, M. Tsujimoto, S. Isoda, F. Kim, K. Kamei, R. A. Fischer, S. Kitagawa and S. Furukawa, J. Am. Chem. Soc., 2013, 135, 10998-11005.

25 P. D. C. Dietzel, B. Panella, M. Hirscher, R. Blom and H. Fjellvag, Chem. Commun., 2006, 959-961.

26 S. R. Caskey, A. G. Wong-Foy and A. J. Matzger, J. Am. Chem. Soc., 2008, 130, 10870-10871.

27 X. D. Liu, H. Y. Zhang, K. Lu and Z. Q. Hu, J. Phys.: Condens. Matter, 1994, 6, L497-L502.

28 S. A. FitzGerald, B. Burkholder, M. Friedman, J. B. Hopkins, C. J. Pierce, J. M. Schloss, B. Thompson and J. L. C. Rowsell, J. Am. Chem. Soc., 2011, 133, 20310-20318.

29 N. L. Rosi, J. Kim, M. Eddaoudi, B. Chen, M. O'Keeffe and O. M. Yaghi, J. Am. Chem. Soc., 2005, 127, 1504-1518.

30 S. Oswald and W. Brückner, Surf. Interface Anal., 2004, 36, 17-22.

31 M. Uchiyama and A. A. Stone, Environ. Sci. Technol., 2006, 40, 3515-3521.

32 Y. Teki, M. Shirokoshi, S. Kanegawa and O. Sato, Eur. J. Inorg. Chem., 2011, 3761-3767.

33 H. Deng, S. Grunder, K. E. Cordova, C. Valente, H. Furukawa, M. Hmadeh, F. Gándara, A. C. Whalley, Z. Liu, S. Asahina, H. Kazumori, M. O'Keeffe, O. Terasaki, J. F. Stoddart and O. M. Yaghi, Science, 2012, 336, 1018-1023.

34 P. D. C. Dietzel, Y. Morita, R. Blom and H. Fjellvag, Angew. Chem., Int. Ed., 2005, 44, 6354-6358. 